\title{
Postkolonialismus und Interkulturelle Theologie in Deutschland: Einleitende Verortungen
}

\author{
Britta Konz und Bernhard Ortmann
}

Infolge von Migration und Globalisierung erhalten postkoloniale Theorie und Interkulturelle Theologie aktuelle Relevanz und Brisanz. ${ }^{1}$ Während in den 199oer Jahren postkoloniale und interkulturelle Fragen und Theorien im deutschsprachigen Raum weitgehend von akademischem Interesse waren, ist die Frage nach dem gesellschaftlichen Umgang mit dem ,Fremden' nun ins Zentrum des öffentlichen Interesses gerückt: Gleichsam ,leibhaftig‘ steht sie heute in Form geflüchteter Menschen einer breiten Öffentlichkeit vor Augen. Zwar werden seit jeher Grenzen überschritten, um bessere Lebensbedingungen $\mathrm{zu}$ finden, und auch der Anteil der migrierenden Weltbevölkerung ist seit den 196oer Jahren annähernd gleich geblieben; dennoch deuten die Entwicklungen der letzten Jahre darauf hin, dass wir uns in

1 Für im deutschsprachigen Raum etablierte Einführungen in den Postkolonialismus vgl. INA Kerner, Postkoloniale Theorien zur Einführung, Hamburg 2012; María do Mar Castro Vare la/Nikita Dhawan, Postkoloniale Theorie. Eine kritische Einführung, Bielefeld ${ }^{2} 2015$; Julia Reuter/Alexandra Karentzos (Hg.), Schlüsselwerke der Postcolonial Studies, Wiesbaden 2012. Zur Interkulturellen Theologie vgl. VolKer KüSter, Einführung in die Interkulturelle Theologie, Göttingen 2011; KLAUS Носк, Einführung in die Interkulturelle Theologie, Darmstadt 2011; Henning Wrogemann, Lehrbuch Interkulturelle Theologie/ Missionswissenschaft 1-3, Gütersloh 2012, 2013, 2015; DE RS., Missionswissenschaft unterwegs. Interkulturelle und interreligiöse Perspektiven, Leipzig 2017. Einführend zur Verbindung beider Bereiche im Rahmen einer Postkolonialen Theologie vgl. Andreas Nehring/ Simon Tielesch (Hg.), Postkoloniale Theologien. Bibelhermeneutische und kulturwissenschaftliche Beiträge, ReligionsKulturen 11, Stuttgart 2013; Sebastian Pittl (Hg.), Theologie und Postkolonialismus. Ansätze - Herausforderungen - Perspektiven, Weltkirche und Mission 10, Regensburg 2018; Michael Nausner, Postkoloniale Theologien, in: VF 57 (2012), 117-130; JUdith GRUBER, Intercultural Theology. Exploring World Christianity after the Cultural Turn, Göttingen 2018. Speziell zu Deutschland vgl. Andreas Ne Hring/SimoN Wiesgickl (Hg.), Postkoloniale Theologien II. Perspektiven aus dem deutschsprachigen Raum, Stuttgart 2017; Michael Nausner, Koloniales Erbe und Theologie. Postkoloniale Theorie als Ressource für deutschsprachige Theologie, in: Judith Gruber (Hg.), Theologie im Cultural Turn. Erkenntnistheologische Erkundungen in einem veränderten Paradigma, Frankfurt a.M. 2013, 131-150. Bezüglich der akademischen Rezeption postkolonialer Theorien im deutschsprachigen Raum benennt Michael Nausner außerdem folgende Personen als „herausragend“: María do Mar Castro Varela, Kien Nghi Ha, Encarnación Gutiérrez Rodríguez und Hito Steyerl, vgl. Michael Nausner, Die langen Schatten der Nofretete. Postkoloniale Theorie und Theologie in Deutschland, in: $\operatorname{Conc}(D) 49$ (2013), 200-209, 201, 208, Anm. 5. 
einem ,Zeitalter der Migration befinden. ${ }^{2}$ Neben dem globalen Ausmaß heutiger Migrationsbewegungen und deren erheblichen wirtschaftlichen und sozialen Einflüssen auf Migrationsgesellschaften spricht vor allem der hohe innen- wie außenpolitische Stellenwert, der dem Thema Migration beigemessen wird, für eine solche Deutung. Die Steigerung der politischen Relevanz vor allem in den ,Aufnahmeländern' ist nicht zuletzt mit dem Terrorismusdiskurs verbunden, in dem Zuwanderung mit Gefahr assoziiert wird, wie auch mit der Infragestellung der Grenzregime souveräner Nationalstaaten durch ,irreguläre Migration. Im Gefolge dieser Entwicklungen gewinnt das rechtsnationale Parteienspektrum an Einfluss, während sich gleichzeitig die Medienpräsenz entsprechender Themen erhöht. ${ }^{3}$

Zunehmend formieren sich vor diesem Hintergrund zivilgesellschaftliche Initiativen, die den Theorierahmen des Postkolonialismus mit konkreten gesellschaftspolitischen Anliegen verbinden. Grundlegend ist hierbei die Annahme, dass die bislang öffentlich kaum diskutierten Verstrickungen Deutschlands in die kolonialen Machtstrukturen der Vergangenheit auch heute noch die gesellschaftliche Auseinandersetzung mit dem ,Fremden bestimmen. So strebt beispielsweise das Bündnis Decolonize Bremen an, in der Hansestadt durch öffentlichkeitswirksame Projekte „koloniales Wirken in der Gegenwart sichtbar zu machen und kolonial-rassistische Strukturen unserer Gesellschaft aufzudecken“4. Mit seinem rassismuskritischen Ansatz widersetzt sich das Bremer Bündnis somit auch jenen gesellschaftlichen Kräften, die im ,Fremden' vor allem eine Bedrohung des ,Eigenen` sehen. Die postkoloniale Perspektive dient hier dazu, etablierte Wahrnehmungsraster zu durchbrechen und aufzuzeigen, dass die weltweite gegenseitige Durchdringung verschiedener Kulturen keine neue Entwicklung darstellt, sondern eine Geschichte hat, aus der sich lernen lässt.

Tatsächlich erscheint dieser Lernprozess dringend notwendig: Angesichts weltumspannender Waren-, Verkehrs- und Informationsströme ist das Lokale heute nicht mehr ohne das Globale zu verstehen (wie auch umgekehrt). Die Rede von der ,Glokalisierung' versucht, dieses Phänomen in Worte zu fassen. ${ }^{5}$ Doch auch in glokaler Perspektive bleiben die Ambivalenzen unserer als ,Postmoderne' titulierten Zeit bestehen: Während einerseits Diversity,

2 Vgl. Stephen Castles et Al., The Age of Migration. International Population Movements in the Modern World, Basingstoke u.a. ${ }^{5} 2014$ [1993], 5.7-8.

3 Vgl. a.a.O., $5^{-6}$.

4 Über uns, Website Decolonize Bremen, https://www.decolonizebremen.com/about (eingesehen am 03.08.2017).

5 Vgl. Roland Robertson, Glokalisierung: Homogenität und Heterogenität in Raum und Zeit, in: Ulrich BeCK (Hg.), Perspektiven der Weltgesellschaft, Frankfurt a.M. 1998, 192-220. 
kulturelle und religiöse Vielfalt befürwortet werden, geht andererseits mit der neoliberalen Globalisierung ein Streben nach marktorientierter Gleichförmigkeit einher. ${ }^{6}$ Auch in Bezug auf Religion ist diese Ambivalenz zu beobachten. Neben einer Befürwortung und Gestaltung eines ökumenischen und interreligiösen Dialoges sind gleichzeitig zunehmend exkludierende Fundamentalismen und Dogmatismen zu beobachten. ${ }^{7}$

Spannungsfelder zeigen sich auch in der aktuell in Deutschland geführten Integrationsdebatte: Immer noch wird sie an vielen Stellen „stark normativ geführt und ist von hegemonialen kulturellen Integrationsvorstellungen durchzogen“8. So steht hinter der Verwendung von Begriffen wie „deutsche Leitkultur“, ,jüdisch-christliche Wertegemeinschaft“" oder "Abendland“ laut Viola B. Georgi vielfach die Imagination eines scheinbar etablierten gesellschaftlichen Konsenses. ${ }^{9}$ Mittels „othering“/VerAnderung werden abgrenzende und zumeist negative Unterscheidungspraxen zwischen dem ,Eigenen' und dem ,Fremden` vollzogen: Das ,Andere' ist dabei für das ,Eigene identitätsstiftend und dient der Sicherung gesellschaftlicher Macht- und Herrschaftsverhältnisse. ${ }^{10}$ Insbesondere Migration droht an dieser Stelle zu einem "gesellschaftsstrukturierenden Metanarrativ“" zu werden, das als allgemein gültige Erklärung für gesellschaftliche Missverhältnisse herangezogen wird. ${ }^{11}$ Zudem wird die Integrationsdebatte wie beispielsweise in der PegidaBewegung auch populistisch konturiert. ${ }^{22}$

Diesem „holistischen Kulturkonzept" mit „hegemonialen Unterdrückungsund Entfremdungsdynamiken ${ }^{\prime 13}$ steht ein Kulturkonzept gegenüber, das nicht

6 Vgl. Josef Estermann, Gott ist nicht europäisch und die Theologie ist nicht abendländisch. Eine interkulturelle Dekonstruktion der abendländischen Theologie aufgrund der indigenen andinen Weisheit, in: IKTh 40 (2014), 186-200, 186.

7 Vgl. ebd.

8 Viola B. Georg I, Integration, Diversity, Inklusion. Anmerkungen zu aktuellen Debatten in der deutschen Migrationsgesellschaft, in: DIE Zeitschrift für Erwachsenenbildung 2 (2015), 25-27, 26 .

9 Vgl. ebd.

10 Vgl. Naika Foroutan/Dilek Ikiz, Migrationsgesellschaft, in: Paul Mecheril (Hg.) unter Mitarbeit von Veronika Kourabas und Matthias Rangger, Handbuch Migrationspädagogik, Weinheim/Basel 2016, 138-151, 138. Zur anfänglichen Prägung des Begriffs des Otherings durch eine der herausragenden Vertreterinnen des Postkolonialismus vgl. Gayatri Chakravorty SpIVAK, The Rani of Sirmur. An Essay in Reading the Archives, in: HTh 24(3) (1985), 247-272, 252-257.

11 Foroutan/IKiz, Migrationsgesellschaft, 139.

12 Vgl. Georgi, Anmerkungen, 26.

13 Franz Gmainer-Pranzl, „Theologie interkulturell“: Diskurs des Christlichen im Horizont des Globalen. Forschungsstand - Arbeitsbereiche - neue Perspektiven, in: 
von statischen, abgrenzbaren und eindeutigen Identitäten ausgeht. ${ }^{14}$ Hier wird vorausgesetzt, dass moderne Gesellschaften diverse Formen ethnischer, gesellschaftlicher, nationaler und religiöser Zugehörigkeit hervorbringen und Migration auch positiv als Motor gesellschaftlicher Veränderungs- und Modernisierungsprozesse fungieren kann. ${ }^{15}$ Migration und Globalisierung führen so zu einer zunehmenden Ausdifferenzierung heutiger Gesellschaften, in denen ethnokulturelle Identität einen Teilaspekt „multidimensionaler Diversität ${ }^{\prime 16}$ bildet. Soziallagen, Milieus und weitere Differenzlinien heben sich, so Georgi, „nicht allein entlang einer Trennlinie zwischen Eingewanderten und Mehrheitsbevölkerung" voneinander ab. ${ }^{17}$ Entsprechend muss in Migrationsgesellschaften der gesellschaftliche Konsens über Werte und Orientierungen auf kultureller und politischer Ebene beständig ausgehandelt und re- bzw. neu formuliert werden. ${ }^{18}$ An diesem Aushandlungsprozess sollten alle gleichermaßen beteiligt sein.

Ein postkolonialer Ansatz ermöglicht es nun, die Komplexität und die Ambivalenzen zu berücksichtigen, die das hier gezeichnete Gesellschaftsbild prägen. Schon ein Seitenblick auf einige der prominentesten Konzepte des Postkolonialismus kann das verdeutlichen: Homi Bhabhas Konzept der Hybridität etwa ist hilfreich, um die vielfältigen Positionierungsmöglichkeiten innerhalb von Migrationsgesellschaften und die sich hieraus ergebenden Mehrdeutigkeiten im Blick zu behalten. ${ }^{19}$ Paul Mecheril spricht diesbezüglich von "natio-ethno-kulturellen (Mehrfach-) Zugehörigkeiten“. ${ }^{20}$ Wie dies vor allem Migrantinnen und Migranten erfahren, können potentiell alle Subjekte "mehrfach und ambivalent in den jeweiligen Stratifikationssystemen platziert" werden. ${ }^{21}$ Für sie haben verschiedene, teilweise auch antagonistische Bezugs- und Orientierungsrahmen eine Bedeutung. Für Subjekte

Ders. Et AL. (Hg.), Herausforderungen Interkultureller Theologie, Paderborn 2016, $11-34,17$.

14 A.a.O., 16.

15 Vgl. Brigit Allenbach et Al., Einleitung, in: Dies. (Hg.), Jugend, Migration und Religion. Interdisziplinäre Perspektive, Religion - Wirtschaft - Politik 4, Zürich, 2011, 13-32, 20, u. Foroutan/IKIZ, Migrationsgesellschaft, 139 .

16 Vertovec, zitiert nach Georgi, Anmerkungen, 25.

17 Georgi, Anmerkungen, 25.

18 Vgl. a.a.O., 27.

19 Vgl. Ном К. Внавна, Die Verortung der Kultur. The location of culture, Tübingen 2000; Jonathan Rutherford, The third space. Interview with Homi Bhabha, in: Ders., (Hg.), Identity: community, culture, difference, London 1990, 207-221.

20 Paul Mecheril, Prekäre Verhältnisse - Über natio-ethno-kulturelle (Mehrfach-) Zugehörigkeit, Münster 2003.

21 Allenbach et Al., Einleitung, 20. 
können sich dadurch Handlungsspielräume ergeben, sie können aber auch in Entscheidungssituationen kommen, die sie bewältigen müssen. ${ }^{22}$ Zudem bedarf es auch der Fähigkeit, mögliche Ambivalenzen auszuhalten und auszutarieren.

Ebenso erhellend ist die durch Edward Said angestoßene OrientalismusDebatte über die Frage der interessegeleiteten Konstruktion des ,Orients im westlichen Diskurs der Vergangenheit, bezieht man sie auf die heutige Inszenierung eines ,christlichen Abendlandes' gegenüber der drohenden ,Islamisierung، der deutschen Gesellschaft. ${ }^{23}$ Und auch Gayatri Chakravorty Spivaks viel zitierte Frage nach der Möglichkeit subalterner Artikulation, also des Gehörtwerdens derer, die vom gesellschaftlichen Diskurs ausgeschlossen sind, ist in ihrer Relevanz für aktuelle Fragen der Wahrnehmung und der politischen Repräsentation von Minderheiten und letztlich des menschlichen Zusammenlebens in Migrationsgesellschaften kaum zu überschätzen. ${ }^{24}$

\section{Herausforderung für die Theologie}

Trotz dieses Potentials führen postkoloniale Ansätze in der deutschsprachigen Theologie ein Mauerblümchendasein. ${ }^{25}$ Dabei muss gerade auch die theologische Reflexion der multikulturellen und multireligiösen Lebenswirklichkeit von Migrationsgesellschaften Rechnung tragen. Denn Religion ist, wie aktuelle Beiträge der Migrationspädagogik zeigen, im Kontext von Migrationsgesellschaften zu einer „Arena für Identitätspolitik“ geworden. ${ }^{26}$ Zudem entstehen im Zuge der Globalisierungsprozesse auch neue Formen

\footnotetext{
22 Ebd.

23 Vgl. Edward W. SAID, Orientalismus, Frankfurt a.M. ${ }^{2} 2010$ [12009, engl. Orig.: ${ }^{1} 1978$; ImAn AtтiA (Hg.), Orient- und IslamBilder. Interdisziplinäre Beiträge zu Orientalismus und antimuslimischem Rassismus, Münster 2007; DIEs., Die „westliche Kultur“ und ihr Anderes. Zur Dekonstruktion von Orientalismus und antimuslimischem Rassismus, Bielefeld 2009.

24 Vgl. Gayatri Chakravorty Spivak, Can the Subaltern Speak? Postkolonialität und subalterne Artikulation, Wien/Berlin 2008; EnCARnación Gutiérrez Rodríguez/ Hito Steyerl (Hg.), Spricht die Subalterne deutsch? Migration und postkoloniale Kritik, Münster 2012.

25 Vgl. NAuSnER, Die langen Schatten, 200.

26 Brigit Allenbach et al., Einleitung, 23. Vgl. hierzu Paul Mecheril/Oscar Thomas-Olalde, Die Religion der Anderen. Anmerkungen zu Subjektivierungspraxen der Gegenwart, in: Brigit Allenbach et Al. (Hg.): Jugend, Migration und Religion. Interdisziplinäre Perspektiven, Religion - Wirtschaft - Politik 4, Zürich 2011, 35-66, Ulrike Lingen-Ali/ Paul Mecheril, Religion als soziale Deutungspraxis, in: Österreichisches Religionspädagogisches Forum 24/2 (2016), 17-24.
} 
des Religiösen. ${ }^{27}$ Religiöse Identitätsbildung bringt so teilweise auch politische Grenzziehungen mit sich. Dabei kann jedoch die Art und Weise, wie Abgrenzungen unter Bezug auf Religion vollzogen werden, unterschiedlich und vielfältig sein. ${ }^{28}$ Für Prozesse, bei denen Religion als „natio-ethno-kultureller Zugehörigkeitscode“ zur Unterscheidung von Gruppen genutzt wird, haben Mecheril und Thomas-Olalde den Begriff des "religiösen Otherings" geprägt. ${ }^{29}$ Insbesondere im Hinblick auf den Islam wird Religion dabei „mit Andersheit (Otherness) verbunden“30 und die Angehörigen der „anderen Religion“ stereotypisiert und essentialisiert. ${ }^{31}$ Damit wird ein Paradox produziert: ,Fremdheit und ,Vertrautheit' werden auf scheinbar konstitutive Weise miteinander verwoben, so dass man meint, die Fremden zu kennen, und zu wissen scheint, wie und wer sie sind. ${ }^{32}$ Das ,Wir' behauptet dadurch seine Deutungshoheit und Dominanzkultur. „Darin“, so Mecheril und Thomas-Olalde, „besteht das ,minimale' und zugleich konstitutive Wissensmorphem hegemonialer Diskurspraxen."33 Gleichzeitig wird das demgegenüber konstituierte „natioethno-kulturelle Wir“ oder ,religiöse Wir' zum imaginativen Raum für positive Selbstbeschreibungen.$^{34}$ Es erscheint homogener, als es tatsächlich ist bzw. es wird erst durch die Abgrenzung konstituiert. Damit beraubt sich das so konstituierte ,Wir jedoch seiner Tiefendimension. Es wird verschleiert, dass die deutsche Kultur schon immer heterogen war und von anderen Kulturen und Religionen mitgeprägt worden ist und dies auch einen Reichtum darstellt, der für die Gegenwart erschlossen werden kann. Für die ,Anderen' haben diese exkludierenden Mechanismen des Otherings gravierende Auswirkungen, weil sie die Subjektivierungspraxen von Individuen mitbestimmen und über die Teilhabe an gesellschaftlichen Aushandlungsprozessen und den Zugang zu Ressourcen entscheiden. ${ }^{35}$ Die kulturelle und religiöse „VerAnderungspraxis“ ist damit nicht ,nur' Unterscheidungs-, sondern auch ",asymetrische Markierungsund Abgrenzungspraxis“. ${ }^{36}$

27 Vgl. Allenbach et al., Einleitung, 23.

28 Vgl. Martin Sökefeld, Religion, Grenzen, Identitäten, in: Brigit Allenbach et AL. (Hg.), Jugend, Migration und Religion. Interdisziplinäre Perspektiven, Zürich 2011, 271286, 282.

29 Mecheril/Thomas-Olalde, Religion, 35-66.

30 A.a.O., 46.

31 Foroutan/IKiz, Migrationsgesellschaft, 142.

32 Vgl. Mecheril/Thomas-Olalde, Religion, 51.

33 A.a.O., 51. Im Original kursiv.

34 Vgl. a.a.O., 62.

35 A.a.O., 49 .

36 Foroutan/IKIZ, Migrationsgesellschaft, 142. 
Postkoloniale Kritik kann hierbei vor Augen führen, wie stark auch das theologische Denken und die kirchliche Praxis von sozialen, politischen und kulturellen Interessens- und Machtstrukturen geprägt sind - beleuchtet sie doch „diskursive Machtkonstellationen, Stereotypenbildungen und Identitätskonstruktionen“ kritisch und dekonstruiert sie „mit Blick auf die Verwirklichung einer freien, gerechten und humanen Gesellschaft" ${ }^{\text {}}{ }^{37}$ Es ist besonders diese Sensibilität für Fragen von Macht und Gerechtigkeit, die den postkolonialen Ansatz aus der Fülle der Möglichkeiten, interkulturelle Fragen zu erörtern, herausragen lässt. ${ }^{38}$

Gerade deshalb bedeutet eine postkolonial-kritische Perspektive für die westlich-akademisch geprägte Theologie auch eine Herausforderung. Denn obwohl Theologie immer „im Kontext und mit den Mitteln einer bestimmten Kultur" entsteht und wirkt, postuliert westliche Theologie noch immer zu sehr Allgemeingültigkeit. ${ }^{39}$ Dabei ist inzwischen offensichtlich, dass sie keine Deutungshoheit beanspruchen kann. Schon die demographische Entwicklung des weltweiten Christentums weist darauf hin: Während 1910 noch $80 \%$ der christlichen Weltbevölkerung in Europa und Nordamerika lebten, waren es 2010 nur noch etwa 45\%. Der demographische Schwerpunkt des weltweiten Christentums hat sich somit eindeutig von der nördlichen in die südliche Hemisphäre verlagert. ${ }^{40}$ Dort vollzieht sich auch ein Großteil des Wachstums der pentekostalen Bewegung, deren jährlicher Zuwachs um 2,2\% zudem noch deutlich über der Rate von $0,09 \%$ der gesamten christlichen Weltbevölkerung liegt. ${ }^{41}$ Dennoch ist pentekostale Theologie in westlich-akademischen theologischen Kreisen nach wie vor kaum bekannt und wenig geschätzt, nicht zuletzt wegen ihrer besonderen Art der theologischen Reflexion, die

37 Franz Gmainer-Pranzl, Der „Logos christlicher Hoffnung“ in globaler Verantwortung. Interkulturelle Theologie: historische und hermeneutische Grundlagen, in: IKTh 40 (2014), 129-148, 143 .

38 Nichtsdestotrotz sind auch andere Ansätze vielversprechend und wurden in der Interkulturellen Theologie rezipiert. Besonders hervorzuheben wäre etwa das Konzept der Transkulturalität, vgl. Wolfgang Welsch, Transkulturalität, in: Art. EnzPh 3 (2010), 2771-2777. Zu dessen Rezeption, häufig in Zusammenhang mit postkolonialen Theorien, vgl. Henning Wrogemann, Interkulturelle Theologie und Hermeneutik. Grundfragen, aktuelle Beispiele, theoretische Perspektiven, Lehrbuch Interkulturelle Theologie/Missionswissenschaft 1, Gütersloh 2012, 338-341; Hock, Einführung, 51-52.

39 Vgl. Franz Martin Wimmer, Was heißt Interkulturalität in Forschung und Lehre der Philosophie?, in: Franz Gmainer-Pranzl/Judith Gruber (Hg.) Interkulturalität als Anspruch, Frankfurt am Main 2012, 11-24 hier 11.

40 Todd M. Johnson/Kenneth R. Ross, Preface, in: Dies. (Hg.), Atlas of Global Christianity. 1910-2010, Edinburgh 2009, X.

41 Todd M. Johnson/David B. Barrett/Peter E. Crossing, Status of Global Mission, 2011, in Context of 2oth and 21st Centuries, in: IBMR 35 (2011), 29. 
stärker auf mündliche Überlieferung und spirituelle Erfahrung fokussiert ist. ${ }^{42}$ Gleichzeitig wächst die Zahl pentekostal geprägter ,Migrationsgemeinden in Deutschland, was es zunehmend erschwert, diese Form des christlichen Glaubens als ,exotisch' und irrelevant für hiesige Kontexte abzutun. Eine postkoloniale Perspektive kann hier dazu beitragen, gerade in der Irritation durch das ,Fremde‘ für beide Seiten eine Möglichkeit zu sehen, sich im Spiegel des ,Anderen' kritisch und konstruktiv mit dem ,Eigenen' auseinanderzusetzen. ${ }^{43}$

Der Interkulturellen Theologie kommt die Aufgabe zu, hierfür im theologischen Diskurs Raum zu schaffen und die theologische, historische und kultur-/sozialwissenschaftliche Reflexion der vielfältigen Ausdrucksformen des christlichen Glaubens in ihren verschiedenen Dimensionen anzuregen. Hervorgegangen aus den Fächern der Missions- und der Religionswissenschaft sowie der Ökumenik befasste sie sich von Beginn an mit den theologischen Entwürfen, der Christentumsgeschichte und der kirchlichen Praxis des ,globalen Südens'. ${ }^{44}$ Auch wurden die ersten Schritte hin zu einer Interkulturellen Theologie in den 1970er Jahren in dem Bewusstsein unternommen, dass dem

42 Vgl. Wonsuk Ma/Veli Matti KärkkäInen/J. Kwabena Asamoah-Gyadu, Introduction: Pentecostalism and World Mission, in: DIEs. (Hg.), Pentecostal mission and global Christianity, Regnum Edinburgh Centenary Series 20, Oxford 2014, 1-9, 2. Neuerdings sind vermehrt auch pentekostale Stimmen im akademischen Diskurs zu vernehmen, vgl. etwa Amos Yong, Renewing Christian Theology, Waco/TX 2014. Zur pentekostalen Bewegung als Teil des Weltchristentums vgl. Allan Heaton Anderson, To the ends of the earth. Pentecostalism and the transformation of world Christianity, Oxford u.a. 2013.

43 Für ein Beispiel aus der Missionstheologie vgl. ANdreas Heuser, „Umkehrmission“ Vom Abgesang eines Mythos im Treppenhaus migratorischer Ökumene, in: IKTh 42 (2016), 25-54. Für einige Beispiele aus der kirchlichen Praxis vgl. We rner KaHL, Vom Verweben des Eigenen mit dem Fremden. Impulse zu einer transkulturellen Neuformierung des evangelischen Gemeindelebens, Studien zu interkultureller Theologie an der Missionsakademie 9, Hamburg 2016, http://www.missionsakademie.de/de/pdf/sitma_9. pdf (eingesehen am 19.10.2016). Zu Migrationsgemeinden in Deutschland allgemein vgl. Michael Bergunder/Jörg Haustein (Hg.), Migration und Identität. Pfingstlichcharismatische Migrationsgemeinden in Deutschland, ZMiss.B 8, Frankfurt a.M. 2006.

44 Zur Diskussion um Bezeichnung und Aufgabe der Interkulturellen Theologie siehe die folgende Erklärung sowie die Reaktionen darauf in Mission Studies: The „Religious Studies and Mission Studies" Section of the Academic Association for Theology (WGTh)/ The Administrative Board of the German Association for Mission Studies (DGMW), Mission Studies as Intercultural Theology and its Relationship to Religious Studies, in: MisSt 25 (2008), 103-108. Vgl. auch das Themenheft Was ist Interkulturelle Theologie?, IKTh 40/2-3 (2014), sowie Dirk-MARTin Grube, Zur Definition und wissenschaftstheoretischen Verortung der Interkulturellen Theologie. Kommentar zu den Beiträgen des Themenheftes „Was ist Interkulturelle Theologie?“ (2-3/2014), in: IKTh 41 (2015), 96-115. 
Ende der Kolonialzeit eine ,entkolonisierte Theologie zu folgen habe. ${ }^{45}$ Seit den 199oer Jahren konnte sie so im westlichen theologischen Diskurs auch durchaus zu einer differenzierteren Wahrnehmung beitragen. ${ }^{46}$ Eine interkulturelle oder gar postkoloniale Perspektive auf Theologie als solche etablieren sich bislang jedoch nicht. Vielmehr bestand der bisherige Beitrag der Interkulturellen Theologie eher darin, als Vermittlerin zwischen einer sich als ,klassisch' verstehenden Theologie und anderen als ,kontextuell ' wahrgenommenen Theologien zu fungieren. ${ }^{47}$ Die Rezeption dieser kontextuellen Theologien in der ",nordatlantischen' akademischen Theologie“ erweist sich jedoch immer noch als schleppend. „Der Eurozentrismus feiert neue Urstände“, lautet Volker Küsters ernüchterndes Fazit zur aktuellen Situation. ${ }^{48}$

45 Der Anfang wurde mit der Begründung der dreisprachigen Reihe Studien zur interkulturellen Geschichte des Christentums (Peter Lang) gemacht. Der erste ihrer inzwischen 161 Bände erschien im Jahr 1975, vgl. https://www.peterlang.com/view/serial/IC (eingesehen am 28.08.2017). In den 199oer Jahren kam im deutschsprachigen Raum der Heidelberger Schule des Missionswissenschaftlers Theo Sundermeier eine bedeutende Rolle zu. Für Umfassenderes zur Entstehungsgeschichte vgl. Werner Ustorf, The Cultural Origins of „Intercultural Theology“, in: Richard Friedui (Hg.), Intercultural Perceptions and Prospects of World Christianity, Frankfurt a.M. u.a. 2010, 81-104, 81-83.89-91.

46 Vgl. a.a.O., 83.

47 Vgl. Estermann, Gott ist nicht europäisch, 188; Volker Küster, Von der Kontextualisierung zur Glokalisierung. Interkulturelle Theologie und postkoloniale Kritik, in:ThLZ 134 (2009), 261-278, 277. Einführend zu kontextuellen Theologien vgl. STEPHEN B. Bevans, Models of Contextual Theology, Maryknoll/NY 2009 [Nachdruck v. ${ }^{2} 2002 ;{ }_{1}^{1992}$ ]. Innerhalb der deutschsprachigen Interkulturellen Theologie sind postkoloniale Theorien dagegen durchaus rezipiert worden, Die Deutsche Gesellschaft für Missionswissenschaft etwa betitelte ihre Jahrestagung 2018 „Postkolonialismus - und was dann?“, https:// www.dgmw.org/2018/09/19/postkolonialismus-und-was-dann-dgmw-jahrestagung-2018/ (eingesehen am 07.05.2019). In den Einführungswerken zeigen sich vor allem folgende Schwerpunkte: zum Bereich ,Christentumsgeschichte‘ vgl. Носк, Einführung, Kap. II. 3.4 „Das Ende der Missionsgeschichte? Interkulturelle Geschichte des Christentums als postkoloniales Projekt“ sowie Kap. II. 4.3 "Ein Blick zurück: Mission, Kolonialismus und Dialektik der Christianisierung“; Wrogemann, Missionstheologien der Gegenwart. Globale Entwicklungen, kontextuelle Profile und ökumenische Herausforderungen, Lehrbuch Interkulturelle Theologie/Missionswissenschaft 2, Gütersloh 2013, Kap. III.6.1 "Geschichte der Frauenmission und postcolonial studies“. Zum Bereich ,Kontextuelle Theologien vgl. Küster, Einführung, 63 sowie Kap. §2.5 „Von der Kontextualisierung zur Glokalisierung“. Zum Bereich ,Kulturtheorie‘ vgl. WrogemanN, Hermeneutik, 8497, 146-155 sowie Kap. IV.3 „Postcolonial Turn` - kulturwissenschaftliche Theorien im Überblick“. Zum Bereich ,Theologie der Religionen` vgl. DERS., Theologie Interreligiöser Beziehungen. Religionstheologische Denkwege, kulturwissenschaftliche Anfragen und ein methodischer Neuansatz, Lehrbuch Interkulturelle Theologie/Missionswissenschaft 3, Gütersloh 2015, Kap. IV.3 „Dialog mit wem eigentlich? - Diskurse“ sowie Kap. v.1.4 "Diskurse und komplexe Identitäten - gegen religionstheologische Essentialismen“.

KüsTER, Einführung, 90. 
Zugleich beobachtet er jedoch, wie sich unter dem Einfluss postkolonialer Theorien im Feld der kontextuellen Theologien „der Akzent langsam von der Kontextualisierung zur Glokalisierung verschiebt. ${ }^{49}$ Denn inzwischen lassen kontextuelle Theologien selbst eine interkulturelle Ausrichtung erkennen, etwa wenn sie der vermeintlichen Allgemeingültigkeit westlicher Theologie subalterne Sichtweisen entgegenstellen und nach deren universaler Bedeutung fragen. ${ }^{50}$

In diesem Zusammenhang rückt Interkulturelle Theologie nicht nur als theologische Disziplin, sondern vor allem auch als Perspektive neu in den Fokus. Mit einem neuen „Problem- und Methodenbewusstsein der Theologie als solche" kann sie die gegenwärtige Theologie bereichern, da sie nicht mehr von einem geschlossenen christlichen System ausgeht, sondern interkulturelle und interreligiöse Spannungsfelder in den Blick nimmt. ${ }^{51}$ Wenn Interkulturelle Theologie nicht lediglich als additive Komponente, sondern als integraler Bestandteil der Theologie ernst genommen wird, kann sich zum einen die theologische Materialgrundlage in quantitativer und qualitativer Hinsicht verändern, zum anderen würde die Theologie "neue Interpretationsperspektiven und methodische [...] Zugänge gewinnen“.52 Interkulturelle Theologie intendiert, wie Franz Gmainer-Pranzl aufzeigt, sowohl "multikulturelle Gleichgültigkeit als auch hegemoniale Universalismen" hinter sich zu lassen, Kommunikationsräume zu öffnen und eine Kultur der Anerkennung und des Lernens zwischen unterschiedlichen kulturellen Traditionen zu ermöglichen. Ziel dieser Kommunikation ist kein neues festgelegtes Deutungssystem, sondern ein bleibendes Aushandeln, im Sinne eines „work in progress“, das auf Verständigung und Konsens zielt, aber auch Unterschiedlichkeiten aushält. ${ }^{53}$ Ein durch den Postkolonialismus geschärftes Bewusstsein für die Grenzbereiche menschlichen Zusammenlebens, für die Trennlinien, die Zwischenräume und die Machtgefälle, die Gesellschaften strukturieren,

49 A.a.O., 92.

5o Ausführlicher zum Wandel kontextueller Theologien und Interkultureller Theologie unter dem Einfluss des Postkolonialismus vgl. Küster, Kontextualisierung. Weiterführend dazu vgl. Bernhard Ortmann, Dynamisierendes Dilemma? Interkulturelle Theologie, Kosmopolitismus und Mission zwischen Universalität und Partikularität, in: Jutta Koslowski/Andreas Krebs (Hg.), Mission zwischen Proselytismus und Selbstabschaffung, ÖR.в 115, Leipzig 2017, 169-182.

51 Franz Gmainer-Pranzl et al., Vorwort, in: Dies. (Hg.), Herausforderungen Interkultureller Theologie, Beiträge zur Komparativen Theologie 26, Paderborn 2016, 7-8, 7 .

52 Reinhold Bernhardt, Interkulturelle Theologie - Ihre Programmatik in systematischtheologischer Perspektive, in: IKTh 40 (2014), 149-172, 151-152.

53 Gmainer-Pranzl, „Theologie interkulturell“, 15. 
erscheint für ein solches Unterfangen kaum verzichtbar. Interkulturelle und Postkoloniale Theologie wären dann kaum noch voneinander zu trennen oder, wie Andreas Nehring es ausdrückt: „Postkoloniale Theologie ist interkulturelle Theologie par excellence. ${ }^{45}$

Für die Theologie ergibt sich aus diesen vielfältigen Überlegungen die Herausforderung, eine Grundeinstellung der ständigen Bewegung einzunehmen, bei der eine Haltung selbstkritischen Denkens über Dominanzstrukturen eingenommen wird. ${ }^{55}$ In diesem Prozess dürfen Standpunkte eingenommen werden, die sich aber immer wieder konstruktiv möglicher Kritik stellen. „Kritik“, so Mecheril und Thomas-Olalde, „ist damit als eine Bewegung zu verstehen, als Projekt einer offenen, kreativen, spannungsvollen Praxis, die die Bedingungen eines anderen, allgemein ,besseren' Lebens zu erkun-

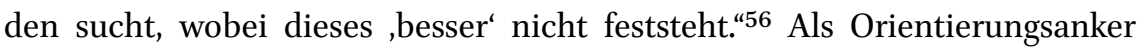
für einen solchen diskursiven interkulturell-theologischen Ansatz können Grundaxiome des christlichen Glaubens, wie Gerechtigkeit und Parteilichkeit für Marginalisierte dienen. Erst wenn kritisch reflektiert wird, welche Literatur rezipiert und welche Stimmen für akzeptabel gehalten werden, kann der volle theologische Reichtum erschlossen werden. Hierzu gehört auch die Einsicht, dass Theologie erst dann zu ihrem Proprium findet, wenn Ansätze der Interkulturellen Theologie als gleichberechtigte Stimme wahrgenommen werden. Darüber hinaus müssen auch Konsequenzen für die (religiöse) Bildung und Lehrerbildung gezogen werden, die die Einsichten postkolonialer Kritik und Interkultureller Theologie in den Diskurs über religiöse Identitätsbildung und den schulischen Unterricht einbeziehen und dazu ermutigen, von denen zu lernen, deren Wissen wir geschichtlich bislang für nichtig gehalten haben.

\section{Bibliographie}

\section{Literatur}

Allenbach, Brigit et Al., Einleitung, in: Dies. (Hg.), Jugend, Migration und Religion. Interdisziplinäre Perspektive, Religion - Wirtschaft - Politik 4, Zürich 2011, 13-32.

Anderson, Allan Heaton, To the ends of the earth. Pentecostalism and the transformation of world Christianity, Oxford u.a. 2013.

54 Andreas Nehring, Die Seite der Schriftleitung, in: ZMiss 38 (2012), 2-3, 2.

55 Vgl. Paul Mecheril/Oscar Thomas-Olalde, Kritik, in: Paul Mecheril (Hg.), Handbuch Migrationspädagogik, Weinheim/Basel 2016, 499.

Ebd. 
Aтt IA, I mAn (Hg.), Orient-und IslamBilder. Interdisziplinäre Beiträge zu Orientalismus und antimuslimischem Rassismus, Münster 2007.

Atтia, Iman (Hg.), Die „westliche Kultur“ und ihr Anderes. Zur Dekonstruktion von Orientalismus und antimuslimischem Rassismus, Bielefeld 2009.

Bergunder, Michael/Jörg Haustein (Hg.), Migration und Identität. Pfingstlichcharismatische Migrationsgemeinden in Deutschland, ZMiss.B 8, Frankfurt a.M. 2006.

Bernhardt, Reinhold, Interkulturelle Theologie. Ihre Programmatik in systematisch-theologischer Perspektive, in: IKTh 40 (2014), 149-172.

Bevans, Stephen B., Models of Contextual Theology, Maryknoll/NY 2009.

Внав на Ном . K., Die Verortung der Kultur. The location of culture, Tübingen 2000.

Castles, Stephen et Al., The Age of Migration. International Population Movements in the Modern World, Basingstoke u.a. $1993{ }^{5} 2014$.

Castro Varela, María do Mar/Nikita Dhawan, Postkoloniale Theorie. Eine kritische Einführung, Bielefeld ${ }^{2} 2015$.

Estermann, Josef, Gott ist nicht europäisch und die Theologie ist nicht abendländisch. Eine interkulturelle Dekonstruktion der abendländischen Theologie aufgrund der indigenen andinen Weisheit, in: IKTh 40 (2014), 186-200.

Foroutan, Naika/Dilek Ikiz, Migrationsgesellschaft, in: Paul Mecheril (Hg.) unter Mitarbeit von Veronika Kourabas und Matthias Rangger, Handbuch Migrationspädagogik, Weinheim/Basel 2016, 138-151.

Georgi, Viola B., Integration, Diversity, Inklusion. Anmerkungen zu aktuellen Debatten in der deutschen Migrationsgesellschaft, in: DIE Zeitschrift für Erwachsenenbildung 2 (2015), 25-27.

Gmainer-Pranzl, Franz, Der „Logos christlicher Hoffnung“ in globaler Verantwortung. Interkulturelle Theologie: historische und hermeneutische Grundlagen, in: IKTh 40 (2014), 129-148.

Gmainer-Pranzl, Franz, „Theologie interkulturell“. Diskurs des Christlichen im Horizont des Globalen. Forschungsstand - Arbeitsbereiche - neue Perspektiven, in: DERS. ET AL. (Hg.), Herausforderungen Interkultureller Theologie, Beiträge zur Komparativen Theologie 26, Paderborn 2016, 11-34.

Gmainer-Pranzl, Franz et al., Vorwort, in: Dies. (Hg.), Herausforderungen Interkultureller Theologie, Beiträge zur Komparativen Theologie 26, Paderborn 2016, 7-8.

Grube, Dirk-Martin, Zur Definition und wissenschaftstheoretischen Verortung der Interkulturellen Theologie. Kommentar zu den Beiträgen des Themenheftes „Was ist Interkulturelle Theologie?“ (2-3/2014), in: IKTh 41 (2015), 96-115.

Gutiérrez Rodríguez, Encarnación/Hito steyerl (Hg.), Spricht die Subalterne deutsch? Migration und postkoloniale Kritik, Münster 2012. 
Heuser, Andreas, „Umkehrmission“. Vom Abgesang eines Mythos im Treppenhaus migratorischer Ökumene, in: IKTh 42 (2016), 25-54.

Hock, KLAUS, Einführung in die Interkulturelle Theologie, Darmstadt 2011.

Johnson, Todd M./David B. Barrett/Peter E. Crossing, Status of Global Mission, 2011, in Context of 2oth and 21st Centuries, in: IB MR 35 (2011), 29.

Johnson, Todd M./Kenneth R. Ross, Preface, in: Dies. (Hg.), Atlas of Global Christianity. 1910-2010, Edinburgh 2009, $\mathrm{x}$.

Kahl, Werner, Vom Verweben des Eigenen mit dem Fremden. Impulse zu einer transkulturellen Neuformierung des evangelischen Gemeindelebens, Studien zu interkultureller Theologie an der Missionsakademie 9, Hamburg 2016, http://www .missionsakademie.de/de/pdf/sitma_9.pdf (eingesehen am 19.10.2016).

KERner, InA, Postkoloniale Theorien zur Einführung, Hamburg 2012.

KÜSTER, VOLKER, Von der Kontextualisierung zur Glokalisierung. Interkulturelle Theologie und postkoloniale Kritik, in: ThLZ 134 (2009), 261-278.

KüSTER, Volker, Einführung in die Interkulturelle Theologie, Göttingen 2011.

Lingen-Ali, Ulrike/Paul Mecheril, Religion als soziale Deutungspraxis, in: Österreichisches Religionspädagogisches Forum 24/2 (2016), 17-24.

Ma, Wonsuk/Veli Matti KärkKäınen/J. Kwabena Asamoah-Gyadu, Introduction: Pentecostalism and World Mission, in: DIEs. (Hg.), Pentecostal mission and global Christianity, Regnum Edinburgh Centenary Series 20, Oxford 2014, 2.

Mecheril, Paul, Prekäre Verhältnisse. Über natio-ethno-kulturelle (Mehrfach-) Zugehörigkeit, Münster 2003.

Mecheril, Paul/Oscar Thomas-Olalde, Die Religion der Anderen. Anmerkungen zu Subjektivierungspraxen der Gegenwart, in: BRIG IT ALLENBACH ET AL. (Hg.), Jugend, Migration und Religion. Interdisziplinäre Perspektiven, Religion - Wirtschaft - Politik 4, Zürich 2011, 35-66.

Mecheril, Paul, Kritik, in: Paul Mecheril (Hg.), Handbuch Migrationspädagogik, Weinheim/Basel 2016, 499.

NAUSner, Michael, Postkoloniale Theologien, in: VF 57 (2012), 117-130.

Nausner, Michael, Koloniales Erbe und Theologie. Postkoloniale Theorie als Ressource für deutschsprachige Theologie, in: Judith Gruber (Hg.), Theologie im Cultural Turn. Erkenntnistheologische Erkundungen in einem veränderten Paradigma, Frankfurt a.M. 2013, 131-150.

Nausner, Michael, Die langen Schatten der Nofretete. Postkoloniale Theorie und Theologie in Deutschland, in: Conc(D) 49 (2013), 200-209.

Nehring, ANDreas, Die Seite der Schriftleitung, in: ZMiss 38 (2012), 2-3.

Nehring, Andreas/Simon Tielesch (Hg.), Postkoloniale Theologien. Bibelhermeneutische und kulturwissenschaftliche Beiträge, ReligionsKulturen 11, Stuttgart 2013 . 
Ortmann, Bernhard, Dynamisierendes Dilemma? Interkulturelle Theologie, Kosmopolitismus und Mission zwischen Universalität und Partikularität, in: Jutta Koslowski/Andreas Krebs (Hg.), Mission zwischen Proselytismus und Selbstabschaffung, ÖR.B 115, Leipzig 2017, 169-182.

Pittl, Sebastian (Hg.), Theologie und Postkolonialismus. Ansätze - Herausforderungen - Perspektiven, Weltkirche und Mission 10, Regensburg 2018.

The „Religious Studies and Mission Studies“ Section of the Academic Association for Theology (WGTh)/The Administrative Board of the German Association for Mission Studies (DGMW), Mission Studies as Intercultural Theology and its Relationship to Religious Studies, in: MisSt 25 (2008), 103-108.

Reuter, Julia/Alexandra Karentzos (Hg.), Schlüsselwerke der Postcolonial Studies, Wiesbaden 2012.

Robertson, Roland, Glokalisierung: Homogenität und Heterogenität in Raum und Zeit, in: Ulrich Beck (Hg.), Perspektiven der Weltgesellschaft, Frankfurt a.M. 1998, 192-220.

Rutherford, Jonathan, The third space. Interview with Homi Bhabha, in: DERs.

(Hg.), Identity: community, culture, difference, London 1990, 207-221.

SAID, EdWARD W., Orientalismus, Frankfurt a.M. ${ }^{2} 2010$.

Sökefeld, Martin, Religion, Grenzen, Identitäten, in: Brigit Allenbach et AL. (Hg.), Jugend, Migration und Religion. Interdisziplinäre Perspektiven, Zürich 2011, 271-286.

Spivak, Gayatri Chakravorty, The Rani of Sirmur. An Essay in Reading the Archives, in: HTh 24 (1985), 247-272.

Spivak, Gayatri Chakravorty, Can the Subaltern Speak? Postkolonialität und subalterne Artikulation, Wien/Berlin 2008.

Themenheft Was ist Interkulturelle Theologie?, IKTh 40/2-3 (2014).

Ustorf, Werner, The Cultural Origins of „Intercultural Theology“, in: Richard Friedli (Hg.), Intercultural Perceptions and Prospects of World Christianity, Frankfurt a.M. u.a. 2010, 81-104.

Welsch, Wolfgang, Art. Transkulturalität, in: EnzPh 3 (2010), 2771-2777.

Wimmer, Franz Martin, Was heißt Interkulturalität in Forschung und Lehre der Philosophie?, in: Franz Gmainer-Pranzl/Judith Gruber (Hg.) Interkulturalität als Anspruch, Frankfurt am Main 2012, 11-24.

Wrogemann, Henning, Lehrbuch Interkulturelle Theologie/Missionswissenschaft 1. Interkulturelle Theologie und Hermeneutik. Grundfragen, aktuelle Beispiele, theoretische Perspektiven, Gütersloh 2012.

Wrogemann, Henning, Lehrbuch Interkulturelle Theologie/Missionswissenschaft 2. Missionstheologien der Gegenwart. Globale Entwicklungen, kontextuelle Profile und ökumenische Herausforderungen, Gütersloh 2013. 
Wrogemann, Henning, Lehrbuch Interkulturelle Theologie/Missionswissenschaft 3. Theologie Interreligiöser Beziehungen. Religionstheologische Denkwege, kulturwissenschaftliche Anfragen und ein methodischer Neuansatz, Gütersloh 2015.

Yong, Amos, Renewing Christian Theology, Waco/TX 2014.

\section{Internetquellen}

Decolonize Bremen, Über uns, https://www.decolonizebremen.com/about (eingesehen am 03.08.2017).

Deutsche Gesellschaft für Missionswissenschaft, Postkolonialismus - und was dann? DGMW-Jahrestagung 2018, https://www.dgmw.org/2018/og/19/postkolonialismusund-was-dann-dgmw-jahrestagung-2018/ (eingesehen am 07.05.2019).

Peter-Lang-Verlagsgruppe, https://www.peterlang.com/view/serial/IC (eingesehen am 28.08.2017) 